Background Anaesthesia worsens OSA, and may lead to respiratory and cardiac complications. Three critical incidents have occurred in the Oxford Hospitals in recent years. OSA is very common at around 5-25\%, but half the cases are not diagnosed. So should we screen for OSA pre-operatively?

Audit Over 9 months from July 2015 all patients completed a STOP-BANG questionnaire in a gynaecology pre-operative clinic. Those with snoring and a score of 3 or greater were referred for a sleep study. Data is presented for 102 patients (100 female; 2 transgender) with a mean $\pm \mathrm{SD}$ age of $55.7 \pm 11.4 \mathrm{yrs}$, BMI $35.8 \pm 7.6 \mathrm{~kg} / \mathrm{m}^{2}$ and collar size $40.0 \pm 4.8 \mathrm{~cm}$.

The rate of non-attendance was high at 19/102 (19\%), with those with a lower STOP-BANG score being more likely not to attend. Of those undergoing a sleep study, a new diagnosis was made in 53/83 (52\%) patients. Symptoms and OSA/hypoventilation were sufficient for CPAP to be started in 23 patients and NIV in 1 (29\% of those screened), with positive diagnoses more likely with higher STOP-BANG scores. The median (IQR) time to CPAP set-up was $80(52,100)$ days, thus a substantial proportion of patients had surgery before treatment.

How should we ensure anaesthetic safety for patients at risk of OSA?

Completing a sleep study and establishing OSA patients on CPAP prior to surgery would significantly slow the surgical pathway, and there is no evidence that this would improve outcome. However it seems sensible for anaesthesia of all patients deemed at risk of OSA (STOP-BANG 3+) to be managed with special precautions. We pragmatically recommend that all patients found to have a STOP-BANG score of $5+$ or strong clinical suspicion of OSA, who are undergoing major surgery AND in whom it is reasonable to delay surgery, are referred for a sleep study prior to surgery. Patients with a STOP-BANG of $3+$ not falling into this category should be informed they are at risk of OSA, and advised to seek a referral from their GP to the sleep clinic if they find symptoms of sleepiness troublesome.

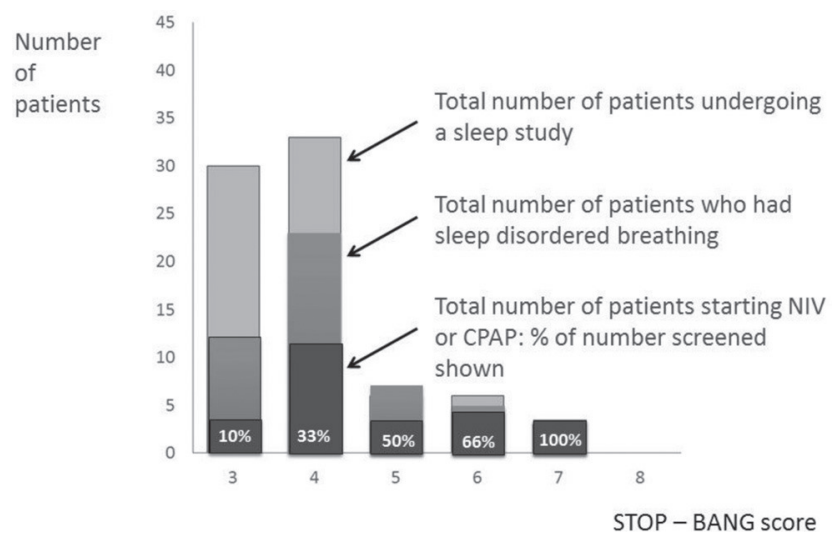

Abstract P68 Figure 1 Data presented by STOP - BANG

\section{P69 EVALUATION OF THE STOPBANG THRESHOLD IN THE PRE-OPERATIVE SCREENING FOR OBSTRUCTIVE SLEEP APNOEA AT SHERWOOD FOREST HOSPITALS FOUNDATION TRUST}

A Reynor, AW Molyneux, SD Tilbrook, RB Dean, E Crookes, J Tansley, NJ Ali. Sherwood Forest Hospitals Foundation Trust, Mansfield, UK

10.1136/thoraxjnl-2016-209333.212
Background Undiagnosed Obstructive Sleep Apnoea (OSA) has been associated with a higher perioperative morbidity and mortality. The aim of this study is to prepare a comprehensive review of the STOPBANG score, a pre-operative screening tool for patients with possible OSA. This study investigates if the current STOPBANG threshold of $\geq 3 / 8$ is appropriate, or if it should be increased to $\geq 5 / 8$ or $\geq 4 / 8$ (with $3 / 4$ in the STOP category) to avoid the unnecessary cancellation/postponement of surgery and inappropriate referrals into the sleep service.

Methods This was a retrospective study of patients referred to the Sleep Service following a positive STOPBANG score $(\geq 3)$. The Research and Development Department of the hospital deemed the study did not require ethical approval. 84 patients were included in the study. The selected patients' case notes were used to review their STOPBANG score, Epworth Sleepiness Score, type of sleep study performed, Oxygen Desaturation Index $(\mathrm{ODI})$, diagnosis and treatment. If the patient had an ODI $\geq 15$, or was successfully started on treatment with a borderline ODI $>5<15$, this was considered an appropriate referral for that threshold.

The sensitivity and specificity of the different STOPBANG thresholds were calculated to assess if the threshold score of STOPBANG $\geq 3$ is appropriate, or if this should be adjusted to more appropriately identify those patients with OSA.

Results For a threshold of $\geq 3$, the sensitivity is very high $(100 \%)$. The sensitivity is decreased for the threshold of $\geq 5$ (71\%), and further decreased for the $\geq 4 / 8$ (3 from STOP) threshold $(53 \%)$. For the $\geq 3$ threshold, the specificity is $0 \%$. The specificity is dramatically increased for the $\geq 5$ threshold (70\%), and highest in the $\geq 4 / 8$ (3 from STOP) category (85\%).

Conclusion The statistical analysis confirms that a threshold of $\geq 3$ has a very high sensitivity, but very low specificity. A threshold of $\geq 5$ has a lower sensitivity however is much more specific and may be a more useful way of identifying the high risk of OSA surgical patient. A change in protocol to $\geq 5$ aims to reduce the unnecessary delay or cancellation of surgery and avoid inappropriate referrals into the Respiratory and Sleep department.

\section{P70 CPAP COMPLIANCE IN BARIATRIC PATIENTS WITH OBSTRUCTIVE SLEEP APNOEA}

${ }^{1}$ PSP Cho, 'A Rainey, 'B Mukherjee, 1,2KK Lee. 'King's College Hospital NHS Foundation Trust, London, UK; 'Division of Asthma, Allergy and Lung Biology, King's College London, London, UK

\subsection{6/thoraxjnl-2016-209333.213}

Introduction CPAP compliance is a challenge in the management of obstructive sleep apnoea (OSA). Pre-operative screening with a sleep questionnaire for OSA followed by sleep studies is common in bariatric services. The King's bariatric service performs overnight pulse oximetry in all patients considered for surgery. Therefore, additional cases may be detected in patients with lower clinical suspicion. We hypothesised that CPAP compliance in bariatric patients with OSA confirmed by routine screening would be lower than that seen in patients referred to the sleep clinic for suspected OSA.

Method Case records of all bariatric patients screened for OSA over a 16-month period were reviewed, and CPAP compliance data retrieved for those with confirmed OSA who commenced CPAP therapy. Retrospective case-control analysis was made against 50 randomly selected patients from the sleep clinic that commenced CPAP for confirmed OSA within the same study 
Abstract P70 Table 1 Baseline and compliance data of bariatric and sleep patients. Data presented as median (inter-quartile range) or n(\%). BMI: body mass index; ODI: overnight desaturation index; ESS: Epworth Sleepiness Score.

\begin{tabular}{lccc}
\hline & Bariatric (n= 49) & Sleep (n= 50) & p-value \\
\hline Age & $49(42-52)$ & $56(44-63)$ & 0.012 \\
Gender (F:M) & $29: 20$ & $20: 30$ & 0.056 \\
BMI (kg/m $)$ & $49.9(46.4-55.9)$ & $42.3(33-48.5)$ & $<0.001$ \\
$\begin{array}{l}\text { Baseline ODI (Average } \\
\text { dips/hour) }\end{array}$ & $31.1(22.3-43.7)$ & $41.0(21.5-61.6)$ & 0.280 \\
$\begin{array}{l}\text { Baseline ESS } \\
\text { Proportion of patients }\end{array}$ & $12(8-15)$ & $11(7.5-15)$ & 0.504 \\
$\begin{array}{l}\text { with } \geq 75 \% \text { of nights } \\
\text { CPAP use }\end{array}$ & $21(42.9 \%)$ & $25(50.0 \%)$ & 0.476 \\
\hline $\begin{array}{l}\text { Proportion of patients } \\
\text { with mean use of } \geq \mathbf{4}\end{array}$ & $20(40.9 \%)$ & & \\
hours per night & & & 0.087 \\
\hline
\end{tabular}

period. CPAP compliance at 4 weeks was compared between the two groups.

Results 409 patients were screened through the bariatric pathway during the study period. $49(12.0 \%)$ patients were diagnosed with OSA and were commenced on CPAP. Baseline characteristics of the bariatric and sleep clinic groups are shown in Table 1. There was no significant difference between gender, baseline overnight desaturation indices and baseline Epworth Sleepiness Scale scores between the two groups.

21 (42.9\%) patients in the bariatric group used their CPAP for $\geq 75 \%$ of nights within the 28 -day period compared with 25 $(50.0 \%)$ patients in the sleep clinic group; $p=0.48$. There was a trend to significance for proportion of patients who used CPAP for mean $\geq 4$ hours per night (20 (40.9\%)) patients in the bariatric group vs $29(58.0 \%)$ patients in the sleep clinic group; $p=0.087)$. Conclusion Our study suggests that the number of patients who are compliant with CPAP at mean use of $\geq 4$ hours per night may be lower in those with confirmed OSA found on routine screening within a bariatric pathway than in patients referred to a sleep clinic, but our finding was limited by the small sample size. Future study to investigate this trend and its underlying causes could improve the success of intervention in the bariatric patients.

\section{P71 CAN POSTURAL OSA BE IDENTIFIED FROM OXIMETRY ALONE?}

${ }^{1}$ A Johar, ${ }^{1} \mathrm{CD}$ Turnbull, ${ }^{2}$ JR Stradling. 'Oxford Centre for Respiratory Medicine, Oxford University Hospitals NHS Foundation Trust, UK; ${ }^{2}$ NIHR Oxford Biomedical Research Centre, University of Oxford, UK

\subsection{6/thoraxjnl-2016-209333.214}

Introduction Positional treatments have a long history of usage in obstructive sleep apnoea (OSA). Recent developments of more sophisticated therapies reported good response in patients with an AHI of $<60$ and predominately supine OSA (arbitrarily defined as total AHI: non-supine AHI $\geq 1.5) .{ }^{1,2}$ We hypothesised that patients with mild to moderate OSA usually have periods both with and without OSA, and that the OSA periods will be mainly due to supine OSA. Such patients might therefore be trialled on positional therapies without specific assessment of posture.

Methods Patients included had OSA and underwent an in-hospital respiratory sleep study between May- July 2016. Sleep studies included video review for identification of supine sleep. The oxygen desaturation index $>4 \%$ (ODI) was recorded in supine and non-supine positions. The ratio of the total to non-supine ODI was calculated.

Results 40 patients' sleep studies were suitable for analysis. 7 patients were excluded: 3 due to comorbidities (hypoventilation/ CSA), 3 due to lack of supine sleep ( $<30$ minutes) and one due to having minimal OSA. Patients had a mean age of 53.8 years (SD 12.7) and mean BMI of $34.9 \mathrm{~kg} / \mathrm{m}^{2}$ (SD 9.0). The median supine ODI was significantly higher than the median total ODI (supine ODI 54.3/h, IQR 47.9; total ODI 28.6/h, IQR 34.4; $\mathrm{p}<0.001)$. There was a significant relationship between the ratio of total ODI to non-supine ODI versus the total ODI (Spearman's rho $=0.37, \mathrm{p}=0.02$; Figure 1 ).

Discussion Our data suggests that patients with lower ODIs are more likely to have supine predominant OSA. At ODI values over 40 it is very unlikely that there is a posturally dependant component to a patient's OSA. However, the reverse is not the case; although many patients with low ODIs do have a postural component, many do not. It is therefore necessary to objectively assess the degree of supine predominant OSA in future trials of positional therapies, but patients with ODIs over 40 could be excluded at the outset. 